\title{
SARS-CoV-2 escaped natural immunity, raising questions about vaccines and therapies
}

\author{
SARS-CoV-2 can escape natural immune responses, but can the virus evade monoclonal antibodies and \\ vaccine-mediated immunity?
}

Emanuele Andreano and Rino Rappuoli

T he COVID-19 pandemic has been full of surprises. The causative coronavirus SARS-CoV-2 effortlessly spread across the world for almost a year with only minimal changes to its RNA genome. However, just as vaccines and therapies entered the clinic, viral variants began to stack up, which raised important questions about what might lie ahead as the immune system battles this evolving foe. Now, in Nature Medicine, Chen et al., Wibmer et al., Xie et al. and Planas et al. provide some relevant insights into this ${ }^{1-4}$.

In contrast to the rapid evolution of other RNA viruses, SARS-CoV-2 has low genomic variability because of its proofreading function. Therefore, to try to control the pandemic, researchers rushed to make vaccines and monoclonal antibodies
(mAbs) tailored to the viral sequence initially identified in Wuhan, China. Thanks to incredible technological progress, huge public-sector investments and global scientific collaboration, vaccines with efficacy of up to $95 \%$ and lifesaving mAbs were developed and approved for emergency use, at a speed never achieved before.

Vaccines and $\mathrm{mAbs}$ developed to combat SARS-CoV-2 target the spike protein, which gives the virus its crown-like ('corona') silhouette. The spike protein binds to the receptor ACE2 on human cells at the onset of infection. There are two distinct antigenic 'supersites' on the spike proteinthe receptor-binding domain (RBD) and $\mathrm{N}$-terminal domain (NTD) - that are the target of most neutralizing antibodies. Antibodies directed against the RBD account for $90 \%$ of the neutralizing activity found in the serum of convalescent patients who have recovered from COVID-19, and antibodies directed against the RBD are 10- to 100 -fold more potent than antibodies that recognize the $\mathrm{NTD}^{5,6}$.

By early December 2020, the first approved vaccines and $\mathrm{mAbs}$ were being rolled out, but this ray of hope was clouded by the emergence of multiple worrying reports of mutated viral variants. Some of these rapidly replaced the original virus identified in Wuhan. The variants most in the spotlight are B.1.1.7 (isolated in the UK), B.1.351 (isolated in South Africa) and B.1.1.248 (isolated in Brazil), which have been named 'variants of concern' (VoC). According to the GISAID database, which has gathered over 960,000 complete and

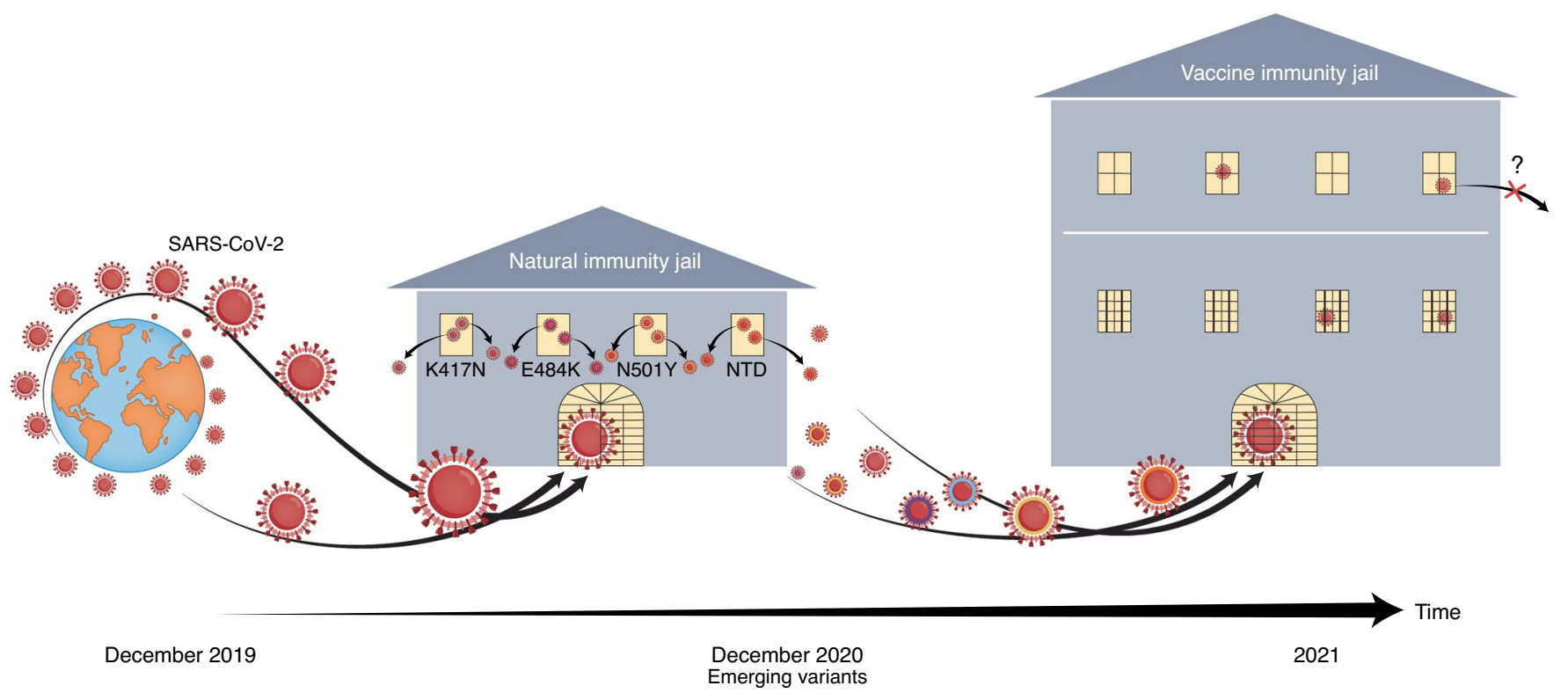

Fig. 1 | Viral variants of SARS-CoV-2 and evasion of immune defenses. In December 2019, SARS-CoV-2 was freely spreading around the world. Natural immunity helped to lock away the virus. However, by December 2020, an emerging viral VoC entered the scene. Characterized by features such as convergent mutations (K417N, E484K and N501Y) encoding alterations to the RBD of the viral spike protein or numerous mutations to the region encoding the NTD of the spike protein, these variants could evade some aspects of natural defenses, such as certain antibody responses. Vaccines and mAbs are under clinical development to fight back against these emerging variants and hopefully provide a more secure way to contain SARS-CoV-2. 
high-coverage SARS-CoV-2 sequences, the VoC account for $37.6 \%(n=361,273)$, $0.83 \%(n=7,969)$ and $0.49 \%(n=4,750)$ of the sequences collected for the UK, South Africa and Brazil, respectively, and their rise continues daily.

The B.1.1.7 variant is characterized by the presence of eight mutations in the region encoding the spike protein, including one mutation (N501Y) on the RBD. This variant is associated with increased mortality in the community of people infected by this lineage ${ }^{7}$. The B.1.351 and B.1.1.248 variants have, among other changes, three key mutations, K417N, E484K and N501Y, in the region encoding the RBD. New viral variants are now reported daily from locations such as the USA, Japan and India, and variants will probably continue to emerge across the globe. An analysis of the viral alterations observed thus far shows that most variants independently acquire the same mutations in the region encoding RBD (K417N, E484K and N501Y), whereas NTD alterations do not seem to converge on a particular set of mutations.

Many of these mutations identified in infected people are also found as escape mutations during in vitro experiments in which the virus or a pseudovirus is incubated with neutralizing mAbs or convalescent serum from people who have recovered from COVID-19; this suggests that these variants are observed because they keep spreading even in populations that have already acquired some immunity after natural infection ${ }^{8}$. The convergence toward similar mutations is probably explained by the need to escape the predominant neutralizing antibody response, which derives mostly from antibodies encoded by the immunoglobulin $\mathrm{G}$ heavy-chain variable germline regions IGHV3-53 and IGHV3-66. Indeed, convalescent serum has limitations in its ability to tackle the B.1.351 and B.1.1.248 variants. Such serum samples can show a four- to ninefold lower neutralization ability when faced with variants than their efficacy against the original SARS-CoV-2 virus identified in Wuhan; moreover, $40-48 \%$ of such serum samples completely lack neutralization ability $y^{1,2,4,10}$. By contrast, the B.1.1.7 variant only moderately affects the neutralization ability of convalescent serum $^{2,10}$.

These variants are having a striking societal effect. A key example is the situation in Manaus, Brazil, where variants have re-infected people and have spread in a population in which over $76 \%$ of people were already SARS-CoV-2 seropositive ${ }^{11}$. The impact of some of the mutations on vaccines and on $\mathrm{mAbs}$ has also been quite disturbing. Three of the four $\mathrm{mAbs}$ that have received emergency use authorization by the US Food and Drug Administration are not effective against the B.1.351 and B.1.1.248 variants, and vaccines have also shown a much lower efficacy against the B.1.1.248 and B.1.351 variants ${ }^{1,2,10}$. Indeed, the majority of antibodies that bind the spike protein's receptor-binding motif (the RBD region that directly interacts with ACE2) are strongly affected by the mutations E484K and K417N, whereas antibodies that bind to a region juxtaposed to the receptor-binding motif are impacted by $\mathrm{N} 501 \mathrm{Y}^{1,2,10}$. Antibodies directed against the NTD are affected by substitutions, deletions and insertions present in different escape variants ${ }^{1,5,10}$. These data confirm that mutations in regions encoding the RBD and NTD enable the virus to escape polyclonal and $\mathrm{mAb}$ responses induced by infection and to conquer some of natural immunity defenses. Fortunately, however, some antibodies directed against the RBD are still able to effectively neutralize most of the $\mathrm{VoC}$, and this is the basis for the development of second-generation therapeutic formulations $s^{5,10}$.

$\mathrm{T}$ cells seem to be less affected by variants. $\mathrm{CD}^{+}$and $\mathrm{CD} 8^{+} \mathrm{T}$ cells target spike-protein epitopes that were shown to be highly conserved among VoC. CD4 ${ }^{+} \mathrm{T}$ cells target conserved epitopes that account for $95.5 \%, 75.3 \%$ and $89.8 \%$ of the epitopes for variants B.1.1.7, B.1.351 and B.1.1.248, respectively, whereas $\mathrm{CD}^{+} \mathrm{T}$ cells target epitopes that are even more conserved, with an average of $96.1 \%$ identity among these three $\mathrm{VoC}^{12}$.

A striking impact on vaccine efficacy against variant B.1.351 has been observed. The efficacy of Novavax vaccine NVX-CoV2373 dropped from $96 \%$ to $48 \%$ (ref. ${ }^{13}$ ); the efficacy of the Johnson \& Johnson vaccine JNJ-78436735 decreased from $85 \%$ to $57 \%$ (ref. ${ }^{14}$ ); and the efficacy of the Oxford-AstraZeneca vaccine ChAdOx1 dropped from $62 \%$ to $10 \%\left(\right.$ ref. $\left.{ }^{15}\right)$. Those drops were matched by a 7 - to 12 -fold decrease in the titer of vaccine-induced neutralizing antibodies against the B.1.351 variant reported for the mRNA-based vaccines, whereas no effect on the neutralization titer was observed for the B.1.1.7 variant ${ }^{3,10}$. In addition, Planas et al. showed that no neutralizing antibodies against the B.1.351 variant were elicited in 15 people $2-3$ weeks after the first dose of the Pfizer-BioNTech mRNA-based vaccine BNT162b2, whereas only $60 \%$ or $77 \%$ of these people developed low titers of neutralizing antibodies 1 week or 3 weeks, respectively, after their second dose $^{4}$. In all cases, the E484K mutation seems to be a major driver of the loss of potency of vaccine-induced neutralizing antibodies, which suggests that a natural infection-like antibody response is induced after vaccination, given that this residue is important for evasion of the antibody response elicited by natural infection and vaccination.

Fortunately, despite the loss of efficacy of all vaccines analyzed, NVX-CoV2373 and JNJ-78436735 showed almost complete protection against severe disease caused by the B.1.351 variant ${ }^{13,16}$. Clinical trials are already underway to test second-generation vaccines and $\mathrm{mAbs}$ in an effort to contain the spread of VoC. As current and future variants could imperil medical interventions, it will be crucial to monitor the effect that new mutations might have on vaccines and antibodies.

Encouraging results suggest that vaccines that contain the spike protein of the B.1.351 variant induce broadly neutralizing antibodies that should contain the spread of VoC. Furthermore, in previously infected people, a single dose of an mRNA vaccine boosts the antibody response by up to 1,000 -fold and propels neutralizing titers of antibodies against VoC to well above the highest titers observed in naive people who received two doses of an mRNA vaccine $e^{17,18}$. This suggests that first-generation vaccines can provide protection against $\mathrm{VoC}$ if they boost an immune system already primed by infection, and possibly can also protect uninfected people who have been primed by two doses of vaccination. Remarkably, cross-neutralizing antibody responses are due almost entirely to RBD-binding antibodies $^{18}$. This can probably be explained by the fact that the sequence encoding the RBD has limited mutation options because the RBD needs to maintain the ability to interact with ACE2, whereas the sequence encoding the NTD has more flexibility to accommodate mutations $s^{4,6,7}$.

This initial phase of the pandemic could be considered like the plot of an action movie that begins with a character (the virus) running freely across the globe, eluding capture until being finally sent to jail (built by natural immunity). However, if this prison is not secure enough (Fig. 1), the virus could escape, aided by certain mutations. Vaccine-induced immunity achieved via spike-protein antigens based on VoC should help ensure those escape routes are securely closed. But the movie has not yet ended. Although we are confident that second-generation vaccines and $\mathrm{mAbs}$ are capable of controlling the virus, whether the virus will continue to surprise by finding new and unexpected escape routes remains an open question. 
Emanuele Andreano' and

Rino Rappuoli (D) 1,2凶

${ }^{1}$ Monoclonal Antibody Discovery Lab, , Fondazione

Toscana Life Sciences, Siena, Italy. ${ }^{2}$ Faculty of

Medicine, Imperial College London, London, UK.

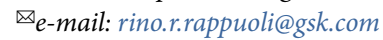

Published online: 10 May 2021

https://doi.org/10.1038/s41591-021-01347-0

References

1. Chen, R. E. et al. Nat. Med. https://doi.org/10.1038/541591-02101294-w (2021).

2. Wibmer, C. K. et al. Nat. Med. https://doi.org/10.1038/s41591021-01285-x (2021)
3. Xie, X. et al. Nat. Med. https://doi.org/10.1038/s41591-021-012704 (2021).

4. Planas, D. et al. Nat. Med. https://doi.org/10.1038/s41591-02101318-5 (2021).

5. Andreano, E. et al. Cell 184, 1-15 (2021).

6. Piccoli, L. et al. Cell 183, 1024-1042.e1021 (2020).

7. Davies, N. G. et al. Nature https://doi.org/10.1038/s41586-02103426-1 (2021).

8. Andreano, E., et al. Preprint at bioRxiv 2020.2012.2028.424451 (2020).

9. Yuan, M. et al. Science 369, 1119-1123 (2020).

10. Wang, P. et al. Nature https://doi.org/10.1038/s41586-021-03398 2 (2021).

11. Sridhar, D. \& Gurdasani, D. Science 371, 230-231 (2021).

12. Tarke, A., et al. Preprint at bioRxiv 2021.02.27.433180 (2021).

13. Novavax. https://ir.novavax.com/node/15661/pdf (11 March 2021).

14. Johnson, C.Y. The Washington Post https://www.washingtonpost. com/health/2021/01/29/covid-vaccine-johnson-and-johnson/ (29 January 2021).
15. Madhi, S. A. et al. N. Engl. J. Med. https://doi.org/10.1056/ NEJMoa2102214 (2021).

16. Johnson \& Johnson. https://www.jnj.com/ johnson-johnson-covid-19-vaccine-authorized-byu-s-fda-for-emergency-usefirst-single-shot-vaccine-in-fight-again st-global-pandemic (27 February 2021).

17. Krammer, F. et al. N. Engl. J. Med. https://doi.org/10.1056/ NEJMc2101667 (2021).

18. Stamatatos, L. et al. Science https://doi.org/10.1126/science. abg9175 (2021)

\section{Competing interests}

R.R. is an employee of GSK group of companies. E.A. and R.R. are listed as inventors of full-length human mAbs described in Italian patent applications 102020000015754, filed on 30 June 2020, and 102020000018955 , filed on 3 August 2020.

\title{
Biomarker approach harnessed in trials of personalized medicine for bladder cancer
}

\begin{abstract}
A multi-arm clinical trial reports results of testing a strategy for the treatment of bladder cancer that uses standard immunotherapy plus biomarker assessment and targeted therapies.
\end{abstract}

\author{
Nazli Dizman, Luis Meza and Sumanta K. Pal
}

\section{I uture trials should incorporate - a biomarker-based design to interrogate these investigational} therapies." Words like this are probably spoken during hundreds, if not thousands, of clinical-research presentations at major medical meetings. In this issue of Nature Medicine, Powles et al. are therefore to be applauded for their work on the BISCAY clinical trial, an adaptive, biomarker-directed platform study ${ }^{1}$. The trial assessed the effect on bladder cancer of using durvalumab, an inhibitor of the checkpoint ligand PD-L1 ('programmed death-ligand 1') for immunotherapy, along with relevant therapy targeted to tumor mutations. This checkpoint inhibitor previously achieved a response rate of $18 \%$ for the treatment of advanced urothelial cancer ${ }^{2}$.

The BISCAY trial evaluated three targeted-therapy approaches and durvalumab use. One treatment was use of the inhibitor AZD4547 to target the growth-factor receptor FGFR3 ('fibroblast growth factor receptor 3') in tumors with mutations in the gene encoding this receptor. Another drug tested was olaparib, which inhibits the DNA-repair enzyme PARP ('poly(ADP-ribose) polymerase'). In some cases of its use, this drug was matched to tumors that had abnormalities in genes encoding components needed for DNA repair. The third drug tested was vistusertib. Vistusertib inhibits the metabolic checkpoint kinase mTOR ('mammalian target of rapamycin') complexes 1 and 2, and this treatment was given if tumors had mutations in genes encoding components of the mTOR-PI3K ('phosphatidylinositol 3-kinase') pathway.

The trial schema was rather complex (Fig. 1a). Some arms were randomized; some were not. People with relevant mutations were enrolled in treatment arms on the basis of their mutational status, except for those in the durvalumab monotherapy arm. The monotherapy arm included a mix of participants, but consisted mainly of those without mutations of interest.

The investigators set the bar high for their study. With an intended sample size of 20 evaluable patients per arm, Powles et al. sought to demonstrate if there was a response rate in excess of $50 \%$ (ref. ${ }^{1}$ ). All arms, which ultimately enrolled between 15 and 29 patients, fell far short of this goal. Leaving aside the obvious caveat of a small sample size, several factors might account for this outcome. For example, the response rate of $31 \%$ for AZD4547 when tested as a monotherapy in the BISCAY trial falls somewhat short of the response rate previously reported (40\%) for the FGFR3 inhibitor erdafitinib ${ }^{3}$, which is approved by the US Food and Drug Administration. Given that the response-rate estimations for the combination therapy of AZD4547 with durvalumab were based on the assumption that AZD4547 can perform as well as erdafitinib, it is therefore not surprising that this combination arm would not meet the ambitious target response rate.

Those in the BISCAY trial who had FGFR3 mutations also had a lower prevalence of positive PD-L1 expression and higher tumor mutational burden than that of those without FGFR3 mutations, which possibly explains why the addition of durvalumab to AZD4547 treatment in the subset with this mutation did not significantly improve the response rate. An inverse correlation between high expression of FGFR3 and T cell depletion is well established for a type of urothelial carcinoma ${ }^{4}$. These results from the BISCAY trial might even call into question the fate 